\title{
Retrieval of temperature and water vapor profiles from radio occultation refractivity and bending angle measurements using an Optimal Estimation approach: a simulation study
}

\author{
A. von Engeln ${ }^{1,2}$ and G. Nedoluha ${ }^{1}$ \\ ${ }^{1}$ Naval Research Laboratory, Remote Sensing Division, Washington, D.C., USA \\ ${ }^{2}$ Institute of Environmental Physics, University of Bremen, Bremen, Germany
}

Received: 28 September 2004 - Published in Atmos. Chem. Phys. Discuss.: 15 March 2005

Revised: 27 May 2005 - Accepted: 10 June 2005 - Published: 7 July 2005

\begin{abstract}
The Optimal Estimation Method is used to retrieve temperature and water vapor profiles from simulated radio occultation measurements in order to assess how different retrieval schemes may affect the assimilation of this data. High resolution ECMWF global fields are used by a state-ofthe-art radio occultation simulator to provide quasi-realistic bending angle and refractivity profiles. Both types of profiles are used in the retrieval process to assess their advantages and disadvantages. The impact of the GPS measurement is expressed as an improvement over the a priori knowledge (taken from a $24 \mathrm{~h}$ old analysis). Large improvements are found for temperature in the upper troposphere and lower stratosphere. Only very small improvements are found in the lower troposphere, where water vapor is present. Water vapor improvements are only significant between about $1 \mathrm{~km}$ to $7 \mathrm{~km}$. No pronounced difference is found between retrievals based upon bending angles or refractivity. Results are compared to idealized retrievals, where the atmosphere is spherically symmetric and instrument noise is not included. Comparing idealized to quasi-realistic calculations shows that the main impact of a ray tracing algorithm can be expected for low latitude water vapor, where the horizontal variability is high. We also address the effect of altitude correlations in the temperature and water vapor. Overall, we find that water vapor and temperature retrievals using bending angle profiles are more CPU intensive than refractivity profiles, but that they do not provide significantly better results.
\end{abstract}

\section{Introduction}

Global Navigation Satellite Systems (GNSS), such as the American Global Positioning System (GPS), the Russian Global Navigation Satellite System (GLONASS), or the future European Galileo system, provide a continuous source of signals at radio frequency. The frequency of these signals is within a range where the impact of clouds can generally be neglected, hence they provide an interesting field for atmospheric remote sensing. Ground-based or spacebased receivers are used to derive either information about the integrated precipitable water vapor (Bevis et al., 1992), or about temperature and water vapor profiles (Fischbach, 1965; Kursinski et al., 1997; Zuffada et al., 1999).

Kursinski et al. (1997) gives an excellent overview of the radio occultation principle and the first proof-of-concept experiment GPS Meteorology (GPS/MET). Measurements can be converted into a profile of bending angles $\alpha$. The bending angle profile can be processed to yield a refractivity profile by applying an Abel transform (Fjeldbo et al., 1971). Following the successful mission of GPS/MET, more satellites carrying radio occultation instruments have been launched, e.g. CHAMP (Wickert et al., 2005), SAC-C (Hajj et al., 2002).

Generally one assumes a spherical symmetric atmosphere around the observation point. Deviations from local spherical symmetry are introduced by atmospheric inhomogeneities and the elliptical shape of the Earth. The elliptical shape of the Earth can be partly compensated for by the introduction of a different Earth's center and a radius of curvature, depending on longitude and latitude (Syndergaard, 1998; Zou et al., 2002). The influence of deviations from a spherical symmetric atmosphere can not be corrected this easily and will in general introduce errors in the retrieved atmospheric profiles, e.g. (Healy, 2001).

Correspondence to: A. von Engeln

(engeln@uni-bremen.de)

(C) 2005 Author(s). This work is licensed under a Creative Commons License. 
Most of the early retrievals of temperature and water vapor profiles from radio occultation measurements were focusing on a direct retrieval approach, thus no a priori (also called first guess, or background) information was incorporated. This allowed for the determination of a dry temperature profile where the amount of water vapor was insignificant. At lower tropospheric altitudes either temperature or water vapor can be determined assuming that the other quantity is well known. Several thousand GPS/MET measurements have been compared with correlative data sets and a statistical agreement within $1 \mathrm{~K}$ mean temperature for an altitude range of $1 \mathrm{~km}$ to $40 \mathrm{~km}$ was found (Rocken et al., 1997).

More recently the process of bending angle or refractivity assimilation by variational systems has been addressed. These systems are defined by the number of dimensions considered, they span the range from 1DVar to 4DVar, e.g. (Palmer et al., 2000; Kuo et al., 2000; Liu et al., 2001; Poli et al., 2002), and allow for the simultaneous determination of temperature, and water vapor profiles. Zou et al. (2000) discusses the advantages and disadvantages of bending angle assimilation using a ray tracer over refractivity assimilation. Several authors have also looked at the possibility to combine radio occultation measurements with another satellite based observation in an assimilation approach, first results were published in von Engeln et al. (2001) on a combination with a microwave limb sounder, more recently combinations with infrared and microwave instruments have followed (Borbas et al., 2003; Collard and Healy, 2003).

In this study we will attempt to quantify, using a simulated atmosphere based upon a high resolution ECMWF dataset, the statistical effect of bending angle or refractivity assimilation with different retrieval setups. Retrieval setups encompass variation of all the error covariance matrices and also the introduction of correlations. These results are intended to provide guidance for those interested in assimilating GPS occultation data in choosing models consistent with their requirements and computational constraints.

Although a fully investigation of the impact of radio occultation in a variational system would require to assimilate radio occultation measurements along with other data, one can also use a simplified 1D setup where only radio occultation data is assimilated. This is justified since radio occultation provides data complement to other satellite or ground based observations. One indicator for this is that even a small number of assimilated radio occultation measurements already show a significant impact in the ECMWF forecast skills (Sean Healy, ECMWF, Reading, personal communication, 2005).

We will estimate the information lost when spherical symmetry is assumed in the assimilation. Calculating a fully 3-dimensional retrieval for a statistically significant number of cases is, however, an extremely computationally expensive task. We will therefore instead provide such an estimate by comparing the accuracy of results obtained from 1dimensional retrievals in a spherically symmetric simulated atmosphere with the accuracy of such retrievals in a fully 3dimensional simulated atmosphere.

Comparing a retrieval based on a spherical symmetric atmosphere with one that includes the full 3-dimensional variability also allows to estimate the impact of a ray tracing scheme in the assimilation that would allow for the modeling of horizontal refractivity gradients along the integration path. These gradients can be important in the lower troposphere. Nevertheless, the inclusion of a ray tracing forward model requires large computer resources, although significant progress has been achieved in this area recently (Syndergaard et al., 2004; Poli, 2004). A 1-dimensional processing would therefore be more desirable.

In addition, we will present the first published comprehensive comparison of retrievals based upon bending angle profile measurements versus retrievals based upon refractivity profile measurements. Again, the choice here is between accuracy and ease of assimilation, with the assimilation of refractivity profiles being somewhat simpler. By using the same retrieval and forward model for bending angles and refractivity assimilation we quantify the impacts of different effects in a uniform set of simulations.

We shall also address the impact of correlations in the background fields as well as in the measurement. A simulation approach is used, where the main advantage over the processing of real radio occultation data is knowledge of the true atmospheric state. Performing this study with real data would required to process a large number of occultations in order to reduce several instrumental and atmospheric effects, e.g. measurement noise, ionospheric influences. It would also require to have a dataset of co-located measurement available to assess the retrieval quality. Also, this approach allows us to impose a spherically symmetric atmosphere when required.

Within this study we shall use simulated radio occultation measurements made from a single LEO satellite for one particular day in 2001 (19 May 2001). This day lies within the time frame of a Naval Research Laboratory assimilation study, focusing on the impact of radio occultation data on a Numerical Weather Prediction (NWP) model. In total 550 GPS occultation events were found for our simulated LEO satellite, and a subset of 110 , chosen to provide a wide range of latitude measurements, was used in this study. Measurements were simulated using a ray tracing or wave optics propagator model and a high resolution European Center for Medium Range Weather Forecasts (ECMWF) dataset. A Optimal Estimation retrieval method was used to derive temperature and water vapor profiles, and a reference pressure.

Our paper is structured as follow: Sects. 2, 3: introduction of the forward and retrieval model applied; Sect. 4: presentation of quasi-realistic retrieval results; Sect. 5: impact of assumed a priori and measurement errors; Sect. 6: impact of assumed a priori and measurement correlations; Sect. 7 : conclusion. 


\section{Forward model setup}

Two forward models have been used in this study, a 3-D Endto-end GNSS Occultation Performance Simulator (EGOPS) to produce quasi-realistic measurements, and a 1-D forward model for the retrieval processing. Within the simulator tool, ECMWF atmospheric analysis fields of 19 May 2001 with 4 time steps, 60 vertical levels, and a T511 horizontal resolution were used (Miller, 1999; Teixeira, 1999; Jakob et al., 2000). The vertical resolution of these fields gradually increases from $20 \mathrm{~m}$ at the surface to about $250 \mathrm{~m}$ at $1 \mathrm{~km}$ altitude, and about $1 \mathrm{~km}$ to $3 \mathrm{~km}$ in the stratosphere. The horizontal resolution is about $0.351^{\circ}$. The upper limit of these fields is around $65 \mathrm{~km}$. We use the MSIS atmosphere for altitudes above $65 \mathrm{~km}$ (Hedin, 1987, 1991).

\subsection{1-D forward model}

The forward model used for the retrieval processing is 1dimensional, and therefore neglects the effects of horizontal variations in the atmosphere. It has already been used for a combination study of a radio occultation with a passive microwave instrument (von Engeln et al., 2001) and a radio occultation sensitivity study (von Engeln et al., 2003a). This type of a 1-dimensional forward model is frequently used in the retrieval of radio occultation data (Kursinski et al., 2000; Palmer et al., 2000; Healy and Eyre, 2000; Palmer and Barnett, 2001; Rieder and Kirchengast, 2001). It shows very good agreement with a 1-dimensional forward model that is implemented in EGOPS and also with the EGOPS ray tracing forward model under spherical symmetry conditions.

The principle equations of the radio occultation 1-D forward model are given in Kursinski et al. (1997). Refractivity $N$ (ignoring the impact of the ionosphere) at each atmospheric level is approximated using the formula from Smith and Weintraub (1953):

$N=77.6 \frac{p}{T}+3.73 \cdot 10^{5} \frac{e}{T^{2}}$

with $T$ atmospheric temperature [K], $p$ atmospheric pressure $[\mathrm{hPa}]$, and $e$ partial water vapor pressure [hPa]. $N$ generally varies between about $350 \mathrm{~N}$ - units in wet regions near the ground to about $0.06 \mathrm{~N}-$ units at $60 \mathrm{~km}$. The refractive index $n$ is calculated at each level from $N$ as:

$n=1+\frac{N}{10^{6}}$

The calculation of the bending angle $\alpha$ as a function of refractive index follows an Abelian integral equation. The forward Abel integral is given by:

$\alpha\left(a_{t}\right)=2 a_{t} \int_{r=r_{t}}^{r=\infty} \frac{1}{\sqrt{(n r)^{2}-a_{t}^{2}}} \frac{\mathrm{d} \ln (n)}{\mathrm{d} r} \mathrm{~d} r$

where the integration is performed through all altitudes $r$ starting from the tangent point $r_{t}$, and the factor 2 results
Table 1. Sampling grid used for the simulated measurements, along with the corresponding bending angle (BA) and refractivity (REF) errors assumed.

\begin{tabular}{clll}
\hline $\begin{array}{c}\text { Height } \\
{[\mathrm{km}]}\end{array}$ & $\begin{array}{l}\text { Sampling } d z \\
{[\mathrm{~km}]}\end{array}$ & $\begin{array}{l}\text { BA Error } \\
{[\mu \mathrm{rad}]}\end{array}$ & $\begin{array}{l}\text { REF Error } \\
{[\text { N-units }]}\end{array}$ \\
\hline $00 \leq \mathrm{z} \leq 25$ & 0.25 & 4.0 & 0.05 \\
$25 \leq \mathrm{z} \leq 40$ & 0.50 & 2.8 & 0.036 \\
$40 \leq \mathrm{z} \leq 60$ & 1.00 & 2.0 & 0.027 \\
\hline
\end{tabular}

from the assumed symmetric atmosphere. The impact parameter $a$ at $r_{t}$ is $a_{t}=n_{t} r_{t}$. Within the radio occultation processing, the inverse of this equation is used to calculate the refractivity profile from the bending angle measurements.

Table 1 summarizes the vertical altitude range and the sampling (reflecting the resolution) of the measurement.

Fresnel diffraction will generally lead to a resolution of about $0.5 \mathrm{~km}$ in the lower atmosphere, if no diffraction correction is performed. Modern Fourier Integral Operator based methods can theoretical improve the vertical resolution of radio occultation data to within $50 \mathrm{~m}$ e.g. Gorbunov and Lauritsen (2004); Jensen et al. (2003), but the current resolution of NWP models is about $250 \mathrm{~m}$ at an altitude of $1 \mathrm{~km}$. Hence this vertical resolution exceeds NWP model resolutions at altitudes of about $1 \mathrm{~km}$ (Teixeira, 1999), although the horizontal resolution is generally poorer due to the limb sounding geometry.

Table 1 lists as well the assumed measurement errors on the bending angle and refractivity profiles, which are inbetween the "proof-of-concept" GPS/MET instrument and expected accuracies of modern receivers (ESA/EUMETSAT, 1998). These errors represent the pure noise part of the measurement, at lower altitudes horizontal inhomogeneities will also contribute to the total error (Palmer et al., 2000). This is address by varying the measurement errors. Useful determination of $\alpha$ and hence $N$ above $60 \mathrm{~km}$ is not possible, due to the poor signal-to-noise ratio of mesospheric radio occultation data.

Refractivity errors are approximated by assuming the same signal-to-noise ratio as given for bending angle measurements. The processing of bending angles to a refractivity profile involves the inverse of Eq. (3), which is essentially a weighted sum of the bending angle measurements. This processing introduces correlations in refractivity measurements at different altitudes (Healy and Eyre, 2000; Steiner and Kirchengast, 2005). The actual size of correlations introduced varies depending on several processing steps, e.g. Rieder and Kirchengast (2001) show very small correlations in the resulting measurement error covariance matrix, while Steiner and Kirchengast (2005) show larger correlations.

These correlations can be included in the measurement error covariance matrix, but for simplicity we will not include these correlations in the retrievals shown here, except when 
explicitly indicated. This represents a simplified setup which allows faster processing since the error covariance matrices are diagonal. For two sets of reasonable error covariance matrices, results for bending angle and refractivity based forward models are very similar. Since different NWP centers have different vertical correlation schemes, we felt that not including any vertical correlation was the most appropriate choice.

\subsection{3 -D ray tracer}

The EGOPS simulator tool (Kirchengast, 1998; Kirchengast et al., 2002) was used to simulate bending angle and refractivity measurements. EGOPS has been extensively tested and validated within simulation studies and real data processing (e.g. Wickert et al., 2004; Gobiet et al., 2004). EGOPS reads in orbital parameters for the GNSS and the LEO satellites and predicts in a first step approximated occultation locations. Our scenario is based on the future EUMETSAT Polar System satellite (also called MetOp 1), the first operational radio occultation instrument planned for launch in 2006, with an orbit altitude of $840 \mathrm{~km}$ and an orbit inclination of $98.7^{\circ}$.

In the second step, EGOPS reads in atmospheric fields and uses a ray tracer or wave optics forward model propagator with sub-millimeter accuracy to calculate the amplitude and phase of the simulated ray path, along with its precise occultation location. The ray tracer includes ionospheric effects simulated by a 3-D ionosphere with a solar activity index of 130 (10.7 cm flux). The tracing terminates when one of the rays hits the Earth's surface, thus not all occultations reach down to the surface. Wave optics calculations, taking Fresnel diffraction into account, are performed without the ionospheric influence, this propagation uses a similar termination criteria as the ray tracer. Almost all 110 simulated ray tracing occultations reach tangent altitudes $\leq 5 \mathrm{~km}$, but only about 20 reach tangent altitudes $<1 \mathrm{~km}$.

The third step of an EGOPS simulation consists of the observation system modeling. This denotes the superposition of all relevant physical and technical influences of the observation system onto the output of the second step, and on the "ideal" orbit data (GNSS and LEO positions and velocities). Relevant influences include effects of precise orbits, simulation of the receiving system, antenna specifications, receiver noise, multipath impacts, and the differencing treatment and clock modeling. All error characteristics follow those of modern receivers (ESA/EUMETSAT, 1998). The output of this third step represents the raw measurement of a LEO GNSS receiver.

The last step of an EGOPS calculation processes the simulated phase and amplitude data (supplemented by the necessary geometrical information) via Doppler shifts and bending angles down to quasi-vertical atmospheric profiles of refractivity, density, pressure, temperature, and humidity. Errors caused by an incorrect impact parameter as discussed by Healy (2001) are thus included in the bending angle and refractivity profiles. Bending angles are essentially calculated following Kursinski et al. (1997), although using an optimized approach (see (Kirchengast et al., 2002) for details). Within this study, bending angle and refractivity profiles were used as quasi-realistic measurements.

\section{Retrieval model}

The inverse model calculates the most likely solution $\hat{\mathbf{x}}$ of the true atmospheric state $\mathbf{x}$. A Bayesian solution based on a linear inverse problem leads to the definition of a cost function; the most likely solution is found by minimizing the cost function, where an iterative approach is applied. The retrieval algorithm used here is based on the Optimal Estimation Method (Rodgers, 2000) or 1-D Var. It uses a priori knowledge on the state of the atmosphere to stabilize the solution. The Optimal Estimation Method is frequently used in the retrieval of profiles from atmospheric measurements, when no full NWP model run is used. Even NWP models rely on 1-D Var schemes for the calculation of certain parameters (S. English, Met Office, Exeter, personal communication, 2005).

The iterative formula to calculate $\hat{\mathbf{x}}$ for the iteration $n+1$ is given as:

$\hat{\mathbf{x}}_{n+1}=\mathbf{x}_{0}+\mathbf{G}_{n}\left[\left(\mathbf{y}-\mathbf{y}_{n}\right)-\mathbf{K}_{n}\left(\mathbf{x}_{0}-\hat{\mathbf{x}}_{n}\right)\right]$

where $\mathbf{x}_{0}$ is the a priori vector from which the iteration starts, $\mathbf{G}_{n}$ the $n$-th iteration of the gain matrix, $\mathbf{y}$ represents the bending angle or refractivity measurement, $\mathbf{y}_{n}$ the forward model output, and $\mathbf{K}_{n}$ the Jacobian.

The Jacobian matrices $\mathbf{K}_{n}$ and $\mathbf{G}_{n}$ are defined as:

$\left.\left.\mathbf{K}_{n} \equiv \frac{\partial F(\mathbf{x})}{\partial \mathbf{x}}\right|_{\mathbf{x}=\mathbf{x}_{n}} \quad \mathbf{G}_{n} \equiv \frac{\partial I(\mathbf{y})}{\partial \mathbf{y}}\right|_{\mathbf{y}=\mathbf{y}_{n}=F\left(\mathbf{x}_{n}\right)}$

where $F(\mathbf{x})$ is the 1-dimensional forward model discussed in the previous section, which creates simulated measurements for any given state $\mathbf{x}$ and $I(\mathbf{y})$ represents the inverse model.

For this study the Jacobian matrix $\mathbf{K}_{n}$ is calculated by perturbing the corresponding retrieval parameter $\mathbf{x}$ of $F(\mathbf{x})$. For the given formulation the matrix $\mathbf{G}_{n}$ can be calculated from:

$\mathbf{G}_{n}=\left(\mathbf{S}_{0}^{-1}+\mathbf{K}_{n}^{T} \mathbf{S}_{y}^{-1} \mathbf{K}_{n}\right)^{-1} \mathbf{K}_{n}^{T} \mathbf{S}_{y}^{-1}$

with the a priori error covariance matrix $\mathbf{S}_{0}$, the error covariance matrix of the measurement $\mathbf{S}_{y}$, and $\mathbf{K}_{n}^{T}$ denoting the transpose matrix of $\mathbf{K}_{n}$.

We characterize the retrieval quality by calculating the standard deviation $S_{t}$ with respect to the true profile at each retrieval altitude for all occultations. The true profile is determined within EGOPS at the actual tangent point of each ray. Results for temperature are expressed in [K], while water vapor results are presented in [\%]. Additionally, an improvement $I$ over the standard deviation of the a priori atmosphere 
wrt the true atmosphere $S_{a}$ is calculated at each retrieval altitude in [\%] by:

$I=100.0 \cdot\left(S_{a}-S_{t}\right) / S_{a}$.

The standard deviation does not give any information about a possible bias in the data, thus biases are separately discussed in the text.

\subsection{Retrieval setup}

The $\hat{\mathbf{x}}$ vector holds the temperature profile between $0 \mathrm{~km}$ and $100 \mathrm{~km}$, the water vapor profile between $0 \mathrm{~km}$ and $20 \mathrm{~km}$, and a reference pressure from which the hydrostatic atmospheric pressure profile is generated. The reference pressure is always retrieved at the lowest retrieval altitude.

Water vapor is expressed in volume mixing ratio (VMR) units, the retrieval itself is performed in $\log (\mathrm{VMR})$. A $\log (\mathrm{VMR})$ is generally used in the Optimal Estimation or 1D Var retrieval from radio occultation data (Healy and Eyre, 2000; Palmer et al., 2000; Poli et al., 2002), since water vapor varies by several orders of magnitude over the considered altitude range.

The vertical retrieval grid is given in Table 2, it is chosen to match the measurement resolution as given in Table 1 in the lower atmosphere, to avoid the introduction of retrieval errors caused by a coarser retrieval grid (von Engeln et al., 2003a). The retrieval steps are gradually increased above $20 \mathrm{~km}$ to compensate for the decreasing signal-to-noise ratio. Retrieval above $60 \mathrm{~km}$ is not possible with the chosen setup, nevertheless the extension of the temperature grid up to altitudes of $100 \mathrm{~km}$ assures that uncertainties in the mesospheric temperatures will be considered in the error budget at lower altitudes. Contributions to the error budget arise from the limb sounding geometry for bending angles since an integration through all altitudes above is performed (see Eq. 3).

Currently processed radio occultation measurements go usually only up to about $30 \mathrm{~km}$ to $35 \mathrm{~km}$ with a direct dry temperature inversion, better removal of ionospheric effect is required above. But the anticipated upper retrieval altitude for the future GRAS instrument (ESA/EUMETSAT, 1998) is expected to be around $50 \mathrm{~km}$, thus we perform retrievals up to $60 \mathrm{~km}$. Water vapor is usually retrieved with a variational approach up to about $10 \mathrm{~km}$, but with future instruments expected to provide water vapor information also higher up, we use an upper altitude of $20 \mathrm{~km}$.

The $\mathbf{S}_{0}$ matrix is generated with a $2.5 \mathrm{~K}$ a priori uncertainty for temperatures up to $20 \mathrm{~km}$ and a linear increase up to $20 \mathrm{~K}$ at $100 \mathrm{~km}$. For water vapor a $40 \%$ uncertainty is generally assumed, and a $1 \%$ error in the reference pressure. These settings are consistent with the capabilities of a NWP model short range forecast calculation (Palmer et al., 2000), except for the $1 \%$ a priori uncertainty for the reference pressure retrieval, but sensitivity of the retrieval to this uncertainty is very low. These errors represent estimated a priori errors, since the actual or true a priori error is generally not known,
Table 2. Vertical retrieval grid.

\begin{tabular}{cll}
\hline Species & Height $[\mathrm{km}]$ & Sampling $d z[\mathrm{~km}]$ \\
\hline Temperature & $00 \leq \mathrm{z} \leq 20$ & 0.25 \\
& $20 \leq \mathrm{z} \leq 30$ & 0.5 \\
& $30 \leq \mathrm{z} \leq 40$ & 1.0 \\
& $40 \leq \mathrm{z} \leq 60$ & 2.5 \\
& $60 \leq \mathrm{z} \leq 100$ & 10.0 \\
Water Vapor & $00 \leq \mathrm{z} \leq 20$ & 0.25 \\
\hline
\end{tabular}

but can be calculated for a simulation study. Temperature and water vapor estimated uncertainties have been varied within this study in order to assess their impact.

The measurement covariance matrix $\mathbf{S}_{y}$ is generated with the errors presented in Table 1. We shall assume that the bending angle measurement error covariance is statistical in nature (i.e., the measurement profile have no systematic bias) (Rieder and Kirchengast, 2001), as mentioned above.

The iterative process in Eq. (4) is run up to iteration 10, but convergence was usually found with less than 4 iterations. Within this process, we use the Levenberg-Marquardt method to find the minimum of the not too non-linear cost function. This method can either start with a steepest descent approach or a Newtonian iteration; test calculations showed that a convergence is faster with an initialization closer to a Newtonian iteration, and this was thus used throughout this study.

\subsection{Simulated measurements and background fields}

We simulate 110 radio occultation measurements by using the 3D forward model on ECMWF analysis data from 19 May 2001. The a priori profile for each measurement is then taken from ECMWF analysis fields from the previous day so that for each retrieval it is $24 \mathrm{~h}$ older than the simulated measurement. The mean tangent point position of the occultation was used to extract the 1-dimensional a priori profiles from the ECMWF fields. The actual a priori error then is the standard deviation of the difference between the temperature and water vapor analysis fields from the two days. If we consider this difference to be representative of the error in the analysis in the absence of the radio occultation measurements, then we can estimate the improvement gained by adding these measurements to the analysis.

A priori profiles are assumed to have no vertical correlations here. By first excluding such correlations we derive two advantages: 1) it is easier to see where the GPS measurements are actually providing direct information and 2) the result is more general, since vertical correlation schemes will vary depending upon who is doing the assimilation (background covariance matrices are generated at NWP centers and depend on the centers variational system setup; thus correlations differ between different centers). The effect of 

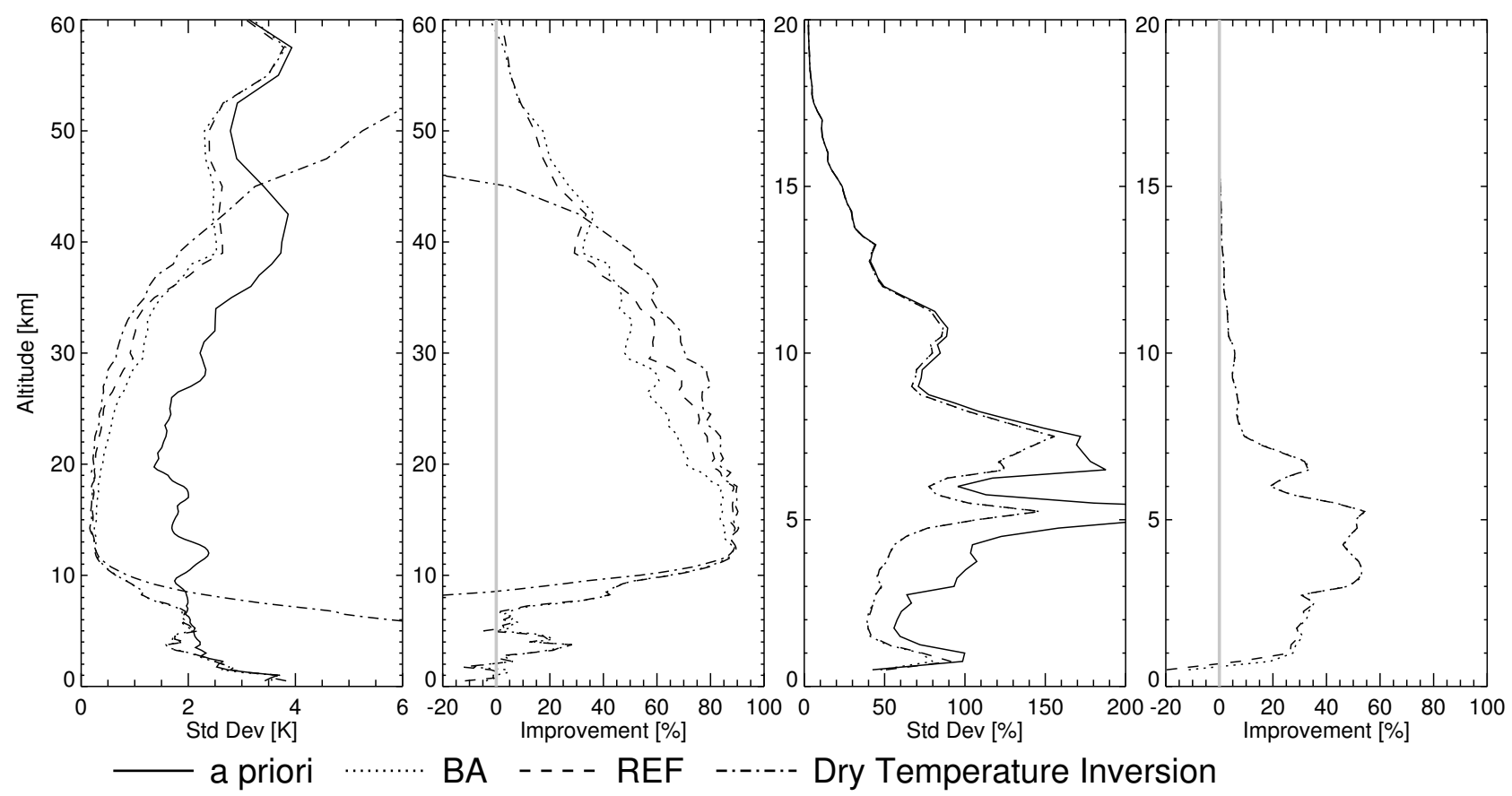

Fig. 1. Standard deviation and improvements of temperature (left) and water vapor (right) wrt true profile, using either bending angles (BA) or refractivity (REF) measurements. The dry temperature inversion, as given by EGOPS is also shown for temperature. Note: different altitude ranges.

a simple correlations on the temperature and water vapor profiles is then evaluated in this study. Generally, the a priori profiles and their error covariance matrices have to be chosen very carefully for a retrieval from real data (Rodgers, 2000), especially a systematic error in the a priori profile will generally lead to the introduction of a bias in the retrieval.

\section{Retrieval results}

Temperature and water vapor profile retrievals from either bending angle or refractivity quasi-realistic measurements were performed for all 110 occultations. A quality check of the retrieved profiles removed 9 occultations, leaving 101 occultations. The standard deviation and the improvement (Eq. 7) over all 101 occultations are presented in Fig. 1. These results are based on ray tracer simulations, wave optics results are very similar and have been omitted for clarity. Multipath generation is possible in ECMWF fields, but EGOPS will tend to stop processing above these areas.

Also shown in Fig. 1 is the dry temperature direct inversion, which uses no a priori data. The processing derives the density from the refractivity data, using the ideal gas law. Pressure can be calculated by integrating the density profile, and temperature follows from the pressure data by invoking the hydrostatic equation. This processing allows the determination of a dry temperature profile in the stratosphere and the upper troposphere. Within the humid areas of the tropo- sphere, either water vapor or temperature has to be estimated in order to determine the other quantity accurately. Disregarding the humid part will lead to a bias in the derived temperature profiles, as can be seen in the large error for the dry inversion near the ground. See Kursinski et al. (1997) for a more thorough description.

The true standard deviation of the temperature a priori data is around $2 \mathrm{~K}$ to $4 \mathrm{~K}$. The true water vapor a priori is provided with a standard deviation of about $50 \%$ to about $200 \%$ in the lowest $10 \mathrm{~km}$. The improvement plots show that the retrieval from refractivity measurements works slightly better with our simplified measurement error covariance setup for temperature in the altitude range of $15 \mathrm{~km}$ to $35 \mathrm{~km}$, yielding improvements over the a priori data of about $85 \%$ to $45 \%$. Above, retrieval from bending angle measurements are slightly better in this simplified setup. The better results are caused by the integration of bending angles through all atmospheric layers above the tangent point, hence all tangent points below a certain altitude provide additional information at this altitude. The impact is mostly found in regions where the temperature retrieval sensitivity decreases.

Improvements in temperature at lower altitudes are only found down to about $7 \mathrm{~km}$. At altitudes below this water vapor begins to significantly affect the measurement. Retrieved temperatures below can even lead to a decrease of the retrieval quality over the a priori data. Although the Optimal Estimation Method succeeds in minimizing the cost function, 

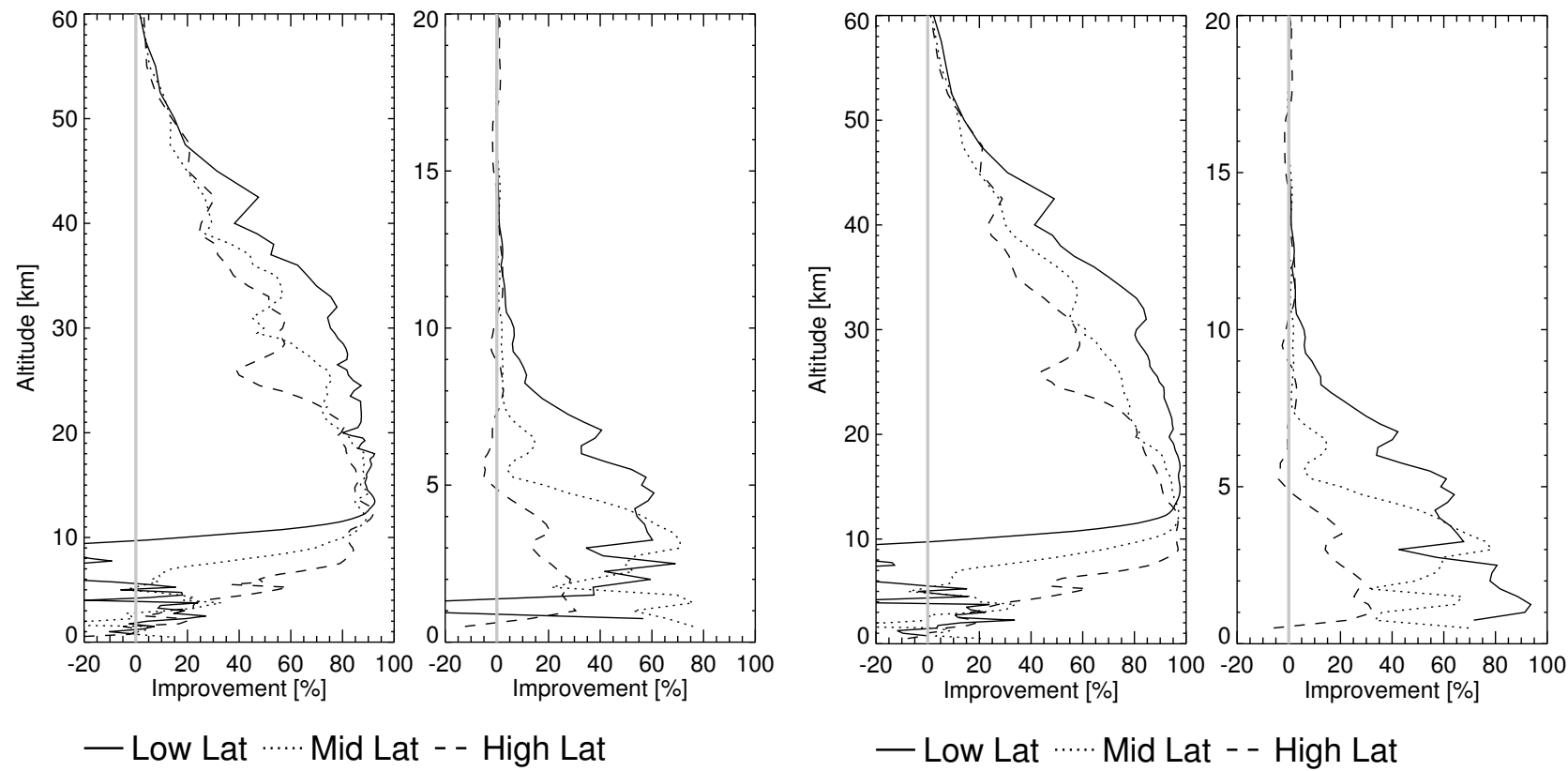

Fig. 2. Latitude separation for improvement of temperature and water vapor wrt true profile for a quasi-realistic (left) and an idealized (right) assimilation, using refractivity measurements. Note: different altitude ranges.

there are occultations where adjustments are made to water vapor to compensate for temperature differences and vice versa. This also occurs in idealized retrievals (see below). A more rigorous quality processing and further research is necessary to filter out retrievals that lead to these erroneous results in the lower troposphere. Similar results are also found in idealized retrievals when a priori errors and actual errors are in agreement.

The dry temperature inversion yields larger improvements over the altitude range of $15 \mathrm{~km}$ to $42 \mathrm{~km}$, but a priori data supports the temperature retrieval of the Optimal Estimation Method above and below, leading to better results. Ideally, the Optimal Estimation Method should yield similar or better results as the direct dry temperature inversion. The results indicate that further optimization of the a priori and measurement errors is possible, as discussed in Sect. 5. Dry temperature retrievals are not influenced by the quality of the a priori data.

Water vapor improvements vary between about $20 \%$ and $40 \%$ for an altitude range of $1 \mathrm{~km}$ to $7 \mathrm{~km}$. The results for retrievals based upon bending angle measurements and refractivity measurements are very similar. Negative improvements are only found below $1 \mathrm{~km}$, but the number of occultations penetrating the lowest $1 \mathrm{~km}$ decreases rapidly, thus results found here are not sufficient to draw conclusions.

The a priori data has a small temperature bias generally below $0.5 \mathrm{~K}$ which is effectively removed by the optimal estimation method above $10 \mathrm{~km}$ since information comes from the unbiased measurement. Below $10 \mathrm{~km}$, the bias is almost unchanged. This can be understood by examining Fig. 1, below $10 \mathrm{~km}$ improvements in temperature are very small, thus the bias is also not removed by the retrieval. The small bias in the water vapor a priori data also follows the water vapor results of Fig. 1 and is reduced for altitudes up to about $7 \mathrm{~km}$.

We find that retrievals using bending angle measurements are very similar to those using refractivity measurements. All further results are thus limited to refractivity measurements, but they have been confirmed with bending angle calculations, where no major differences were found.

All presented results are based on ray tracer simulations with EGOPS, but have also been confirmed with wave optics simulations. Ray tracer calculations give slightly better results in the lower troposphere, since wave optics calculations will introduce a vertical smoothing of the highly variable water vapor field. However, this only reflects the fact that these simulations neglect the effect of diffraction

Figure 2 shows the retrieval from quasi-realistic and idealized refractivity measurements separated by latitude band. The mean latitude $\theta$ of an occultation defines the latitude band as: low latitude $|\theta|<30^{\circ}$, high latitude $|\theta|>60^{\circ}$, and mid-latitudes in between.

The idealized retrieval is performed by assuming the same forward model in the generation of a simulated measurement and within the retrieval model, following Eq. (3). The measurement is free of measurement biases, ionospheric effects, and other observation system influences, thus only Gaussian noise is considered. Furthermore, the temperature, water vapor, and pressure profile are only defined on 


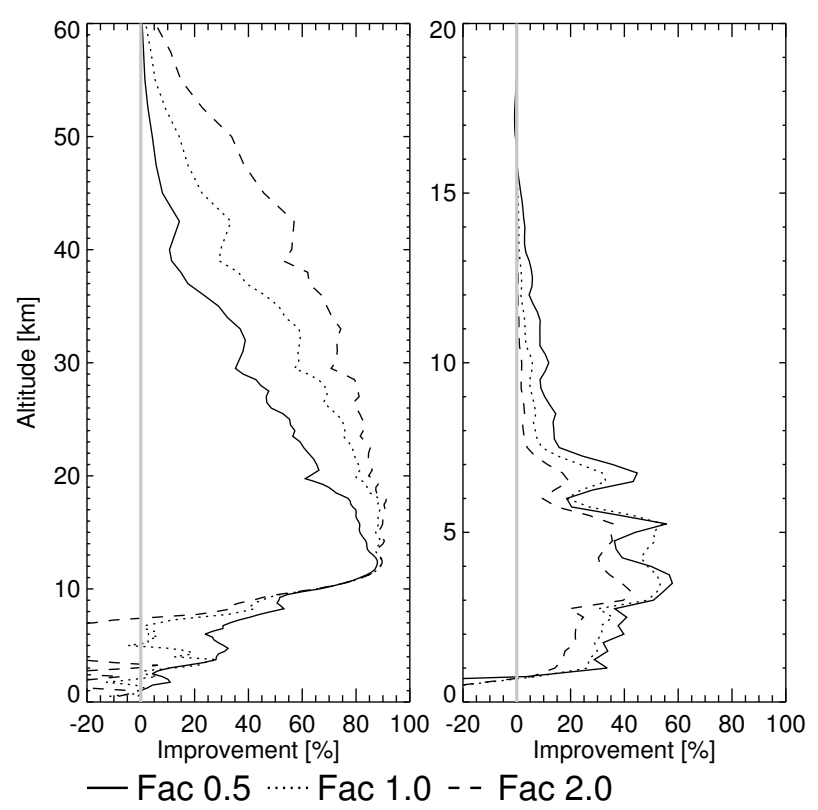

Fig. 3. Impact of temperature a priori error variations on improvement of temperature (left) and water vapor (right) wrt true profile, using refractivity measurements. Note: different altitude ranges.

the actual retrieval grid, representing a 1-dimensional calculation both in the forward and the retrieval model. Thus no fine scale structure, as discussed in von Engeln et al. (2003a), is present.

Although the number of retrievals within a latitude band is only around 25 to 45 , some dominant features can also be reproduced for smaller subsets. Low latitude retrievals from quasi-realistic occultations show larger improvements for temperature in the upper troposphere, and throughout the stratosphere. This is due to higher quality a priori data, since the atmospheric temperature variability at lower latitudes is small. High latitudes show generally the smallest improvements in the upper atmosphere. The impact of water vapor is clearly visible at lower altitudes. Low latitude retrievals start to produce negative improvements at altitude below about $10 \mathrm{~km}$, while mid latitudes and high latitudes yield positive improvements further down. Quasi-realistic and idealized calculations are very similar, the three dimensional structure of the atmosphere degrades the improvement only by a few percent. Hardly any difference is found in the lower troposphere, the three dimensional structure of the atmosphere is not responsible for the degraded improvement here.

The high water vapor concentration at low latitudes yields positive improvements up to about $11 \mathrm{~km}$ for quasi-realistic retrievals. Mid latitude occultations only reach up to about $7 \mathrm{~km}$, and high latitudes up to about $4.5 \mathrm{~km}$. Lower altitudes are highly variable, where negative improvements are found around about $1.0 \mathrm{~km}$ to $1.5 \mathrm{~km}$ for low latitude occultations in a quasi-realistic setup. These could be caused by the sharp water vapor gradient at the planetary boundary layer, as for example discussed in von Engeln et al. (2003b) and by the horizontal inhomogeneities in water vapor. The idealized simulations do not show these negative improvements, indicating that the horizontal variability is mainly responsible. A ray tracer assimilation scheme should thus improve the water vapor retrieval at low latitudes, since it includes the horizontal inhomogeneities (Syndergaard et al., 2004; Poli, 2004). But it also shows that the capabilities of radio occultation observations are mainly limited by the inability to separate water vapor and temperature effectively in the lower troposphere, and not by horizontal inhomogeneities. This is presumably the reason for the ambiguous temperature retrieval results at lower altitudes as presented in Liu and Zou (2003) (Fig. 12, 13). Also, more recently Steiner and Kirchengast (2005) reported similar results, where the improvement in humidity is mainly found at low latitudes.

This limitation is also visible in the main reason for the removal of occultations, as mentioned above: an unreasonable large water vapor or temperature adjustment in an altitude region where the sensitivity to water vapor (temperature) retrieval decreases (increases). All these occultations converge and minimize the difference between the measurement vector and the $1 \mathrm{D}$ forward results. The occultations which have been removed from this study all occurred at or near midlatitudes.

\section{Impact of errors}

The estimated a priori and measurement errors, as given by the covariance matrix $\mathbf{S}_{0}$ and $\mathbf{S}_{y}$, have been varied by factors of 0.5 and 2.0 to assess their impact on the retrieval quality. Figure 3 shows the improvements for a variation in the temperature a priori error only, a higher a priori error will generally put more weight on the measurement, while lower errors will lead to more restrained retrievals.

A higher constraint by the a priori temperature error (Fac 0.5 ) leads to a degradation in retrieval quality at altitudes above about $12 \mathrm{~km}$. A looser constraint improves the retrieval quality at these altitudes, with a very loose a priori yielding the largest improvements (Fac 2.0). Even higher factors of 3.0 and 4.0 show only small further improvements over the Fac 2.0 calculation. Bias removal results at these altitudes also improve with higher a priori errors. Lower altitudes yield some improvements with a more constrained a priori, since temperature adjustments are reduced. Looser a priori constraints yield large negative improvements, the Optimal Estimation Method does not separate temperature and water vapor correctly (Fac 2.0). Water vapor results show similar features, the more constrained temperature leads also to better water vapor retrievals, although variations are small.

Hence temperature a priori error variations show two different effects, at higher altitudes they basically vary the effect of the measurement vs. the a priori error, while at lower 
altitudes they vary the relative constraint on the temperature vs. that on water vapor.

Variation of the water vapor a priori errors will lead to almost identical results in the lower troposphere as the ones presented for temperature, except that the variation factors 0.5 and 2.0 are interchanged (not shown). Higher water vapor a priori errors will lead to better temperature and water vapor retrieval.

The impact of different a priori errors in the lower troposphere can be understood by examining Fig. 1. The true standard deviation of the temperature a priori is around $2 \mathrm{~K}$ in most parts of the troposphere, about the estimated a priori error applied within the Optimal Estimation Method. The true standard deviation of the water vapor a priori is higher than the estimated a priori error within the Optimal Estimation Method. These errors should theoretically match to give optimal results, or the relative weight given to the temperature and water vapor by the a priori error should match. Figure 3 shows the possible optimized retrieval results for the applied setup. Thus, the factor 0.5 results most accurately reflect the relative uncertainties in the water vapor and temperature profiles, and thus give the best results. But practically true standard deviations of the a prioris are not accurately know. This could cause a possible degradation of the temperature retrieval results over the a priori estimate. The bias also shows the highest improvements for an optimized setup.

Estimated measurement errors as given in Table 1 have also been varied by factors of 0.5 and 2.0 to assess their impact on the retrieval quality (not shown). Furthermore, the pure noise part that is super-positioned within the EGOPS simulator has also been varied by these factors. Stratospheric temperature results look very similar to Fig. 3, since variations of the measurement or a priori error shift the impact of measurement and a priori knowledge on the solution. Thus, improved signal-to-noise ratios will lead to higher stratospheric retrieval quality. Below about $12 \mathrm{~km}$ the retrieval is insensitive to the range of estimated measurement errors used here, since at these altitudes the limitations of the retrieval quality are mainly caused by the inability to separate temperature and water vapor effectively. Consequently, water vapor results are also unaffected by the assumed measurement error. Measurement errors around $1 \%$ in refractivity in the lower troposphere, as discussed by Healy and Eyre (2000), do not yield substantially different results in the lower troposphere, improvements in water vapor are still about $20 \%$ for the lowest $5 \mathrm{~km}$. For the bias removal similar results are found, temperature is improved with a lower measurement error in the range of about $20 \mathrm{~km}$ to $40 \mathrm{~km}$, on water vapor this has no effect.

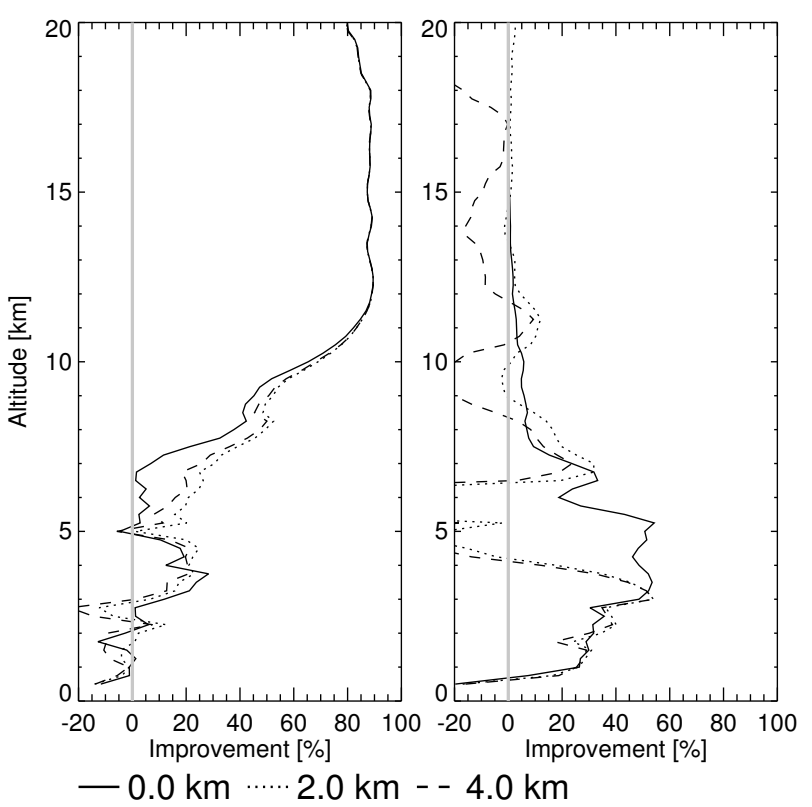

Fig. 4. Impact of correlations in the water vapor a priori on the improvement of temperature (left) and water vapor (right) wrt true profile, using refractivity measurements.

\section{Impact of correlations}

Vertical correlations in the measurements are implicitly introduced when refractivity is used as the measured quantity, but in previous calculations we have set the offdiagonal elements in the covariance matrix $\mathbf{S}_{0}$ and $\mathbf{S}_{y}$ to zero and have therefore not included any explicit vertical correlations. Nevertheless, there clearly are vertical correlations in the atmosphere, and it may be advantageous to include vertical correlations in the a priori.

We test correlation lengths of $2 \mathrm{~km}$ and $4 \mathrm{~km}$ for the temperature and water vapor a priori in order to get some information on the affect of correlations. Correlations are assumed to follow an $1 / e$ function, where correlations between level $i$ and $j$ are calculated as:

$$
\mathbf{S}_{0}[i, j]=\sqrt{\mathbf{S}_{0}[i, i] \cdot \mathbf{S}_{0}[j, j]} \cdot \exp (-|z[j]-z[i]| / c)
$$

with the vertical retrieval levels $z$ and the correlation length $c$.

Correlations of $2 \mathrm{~km}$ and $4 \mathrm{~km}$ were introduced to the temperature a priori covariance matrix (not shown). Effects on the retrieval are similar to the effects shown in Fig. 3 between factors of 1.0 and 2.0, e.g. an improvement of up to $15 \%$ at higher altitudes and a negative improvement at lower altitudes, where both correlation length yield very similar results. Hence, these correlations essentially reduce the weight of the a priori in this setup. The impact of correlations in the water vapor a priori, while leaving temperature uncorrelated, is shown in Fig. 4. 
Although temperature improvements are slightly better in the lower troposphere, retrieval adjustments for water vapor cannot adequately follow the vertically highly variable fields at altitudes above about $3 \mathrm{~km}$, since they are constrained by correlations. Especially retrieval altitudes with a low sensitivity toward water vapor retrieval are affected, while the lowest few kilometers are almost unchanged. These results are improved but do not disappear by the simultaneous introduction of temperature correlations in the a priori covariance matrix.

As mentioned above, the processing from bending angle profiles to refractivity profiles introduces correlations between measurements at different altitudes. The correlations can be modeled by introducing off-diagonal terms in the $\mathbf{S}_{y}$ matrix. Improvements presented so far have assumed a correlation length of $0 \mathrm{~km}$ in the measurement error covariance matrix $\mathbf{S}_{y}$, representing no correlations. Healy and Eyre (2000) assumed a correlation length of $10 \mathrm{~km}$, we used correlations of $2 \mathrm{~km}, 4 \mathrm{~km}$, and $8 \mathrm{~km}$ in $\mathbf{S}_{y}$. Correlations were calculated following Eq. (8), where $\mathbf{S}_{0}$ is replaced by $\mathbf{S}_{y}$.

Temperature retrieval results are degraded by about 10 to $20 \%$ by correlations in the measurement covariance matrix at altitudes above about $12 \mathrm{~km}$ (not shown). Results are hardly altered by the actual correlation length. Correlations as introduced over here will lower the information of the measurement, leading to degraded retrieval results. Thus results are similar to a variation of the measurement errors. The water vapor improvement shows no variations with the correlation. As mentioned above, the water vapor improvement is only influenced by the inability to separate temperature and water vapor effectively.

Retrievals applying also correlations in the temperature and water vapor a priori covariance matrix still show the above mentioned problem with the adjustment of water vapor at higher retrieval levels (Fig. 4).

\section{Conclusions}

A study into the retrieval capabilities of radio occultation data using the Optimal Estimation Method is presented. Quasi-realistic measurements were simulated using a stateof-the-art radio occultation simulator. Ray tracer calculations were used within this study, but wave optics calculations, taking into account Fresnel diffraction, showed no significant difference. Calculations are based on simulations, since small differences between the processing can be determined more accurately. Orbits and assumed errors were generally based on the future GRAS instrument on the EUMETSAT Polar System spacecraft, planned for launch in 2006 (ESA/EUMETSAT, 1998). High resolution ECMWF atmospheric fields were used within the simulation for one particular day in May, 2001. In total, 110 occultations out of a possible 550 for this day were processed to assure a wide variety of atmospheric scenarios. A quality check removed 9 of these occultations, leaving 101 for the presented results.

Assimilation of radio occultation data into a NWP model can either be based on profiles of bending angle or refractivity. Both options are discussed within a 1D Var to 4D Var framework. Refractivity profiles are generated from bending angle profiles and both allow for the inclusion of a nonsymmetric atmosphere. But in a 1-dimensional setup bending angle profiles are more complex to calculate, while refractivity profiles are faster to assimilate. Within this study we either use bending angle or refractivity profiles as input to the Optimal Estimation Method to get information on the best assimilation variable for NWP models, and to test the impact of several retrieval parameter settings. The applied Optimal Estimation Method is a simplified version of an 1D Var algorithm, thus results yield general information about the assimilation of radio occultation measurements.

A simplified setup is used where both measurement error covariance matrices are diagonal, allowing faster processing. A more complex scheme would propagate bending angle errors to refractivity errors, which might introduces correlations in the refractivity matrix, depending on the processing steps. However, our results for bending angles and refractivity are very similar, showing that this simplified choice is valid.

The a priori profiles were also taken from ECMWF fields, they provide temperature information with a standard deviation of around $2 \mathrm{~K}$ to $4 \mathrm{~K}$ for the altitude range $0 \mathrm{~km}$ to $60 \mathrm{~km}$. Water vapor is provided with a standard deviation of about $50 \%$ to about $200 \%$ between $0 \mathrm{~km}$ and $10 \mathrm{~km}$. Retrievals are performed on a $0.25 \mathrm{~km}$ grid up to $20 \mathrm{~km}$ for temperature and water vapor. Above, only temperature is retrieved with the vertical resolution degrading with increasing altitude.

Retrieval results are expressed in improvement over the standard deviation of the true a priori, as given by the ECMWF fields. Temperature improvements vary between about $85 \%$ to $20 \%$ for the altitude range of $15 \mathrm{~km}$ to $48 \mathrm{~km}$. At lower altitudes, improvements are only found down to about $7 \mathrm{~km}$, an altitude where water vapor becomes important. Water vapor improvements are around $20 \%$ to $40 \%$ for an altitude range of $1 \mathrm{~km}$ to $7 \mathrm{~km}$. No pronounced difference between the use of bending angles or refractivity was found.

Results have also been confirmed with ideal retrieval setups, removing horizontal inhomogeneities and reducing the error to Gaussian noise. Thus the major limitation of radio occultation processing in the lower troposphere is the inability to separate the affects of temperature and water vapor effectively.

Temperature improvements have been compared to a dry temperature inversion, which does not use any a priori data. This processing can yield slightly larger improvements within the lower and middle stratosphere if the a priori error of the Optimal Estimation Method are not chosen carefully. 
Much better results are achieved by using the Optimal Estimation Method above and below this altitude range.

A latitudinal separation of the temperature and water vapor improvements shows that temperature for low latitude occultations is affected by water vapor from about $10 \mathrm{~km}$ downwards. Mid-latitudes and high latitudes generally yield temperature improvements further down since less water vapor is present. Water vapor improvements are found up to an altitude of $11 \mathrm{~km}$ for low latitudes, mid-latitudes (high latitudes) reach only up to $7 \mathrm{~km}(4 \mathrm{~km})$. Water vapor retrieval from low latitude occultations are affected by horizontal inhomogeneities at altitudes below about $4 \mathrm{~km}$, visible in a comparison to idealized retrievals. Thus ray tracer calculations are expected to show the highest impact for low latitude water vapor profiles.

Estimated a priori errors of water vapor and temperature have been varied by factors of 0.5 and 2.0 within the Optimal Estimation Method. Altitudes above about $12 \mathrm{~km}$ benefit from a high estimated a priori error in temperature. Lower altitude estimated a priori errors should ideally match the true standard deviation of the a priori profiles with respect to the true one in order to optimize results. Optimized improvements in temperature show an almost linear decrease from $85 \%$ at $11 \mathrm{~km}$ to $0 \%$ at $1 \mathrm{~km}$. Optimized water vapor improvements affect mainly the retrieval at higher altitudes, improvements of around $10 \%$ are still found at $11 \mathrm{~km}$. A mismatch in these errors could even lead to a degradation of the temperature results over the a priori estimate. Variations in the applied measurement error show that a reduction could improve temperature retrieval in the stratosphere. Tropospheric retrieval capabilities are unchanged, the main limitation in the lower troposphere is the inability of the retrieval algorithm to separate water vapor and temperature effectively.

Correlations in the estimated a priori temperature covariance matrix only lead to similar results as an increase in the temperature a priori errors, namely a degradation of the temperature and water vapor retrieval capabilities at lower altitudes, while upper altitudes are improved. Correlations in the a priori water vapor covariance matrix only improve the temperature retrieval slightly, but water vapor improvements are strongly degraded at altitudes above about $3 \mathrm{~km}$. Overall, correlations in the a priori covariance matrix have to be treated with care, and a more conservative setting with no correlations is recommended, at least for the presented setup. Correlations in the refractivity measurement lead to a degradation of the temperature improvement at altitudes above about $12 \mathrm{~km}$. Lower tropospheric water vapor and temperature improvements are unaffected for the above mentioned reasons. Thus, statistically correlations are not important if a proper setup of uncorrelated a priori and measurement error is chosen.

Overall, retrieval from refractivity measurements shows several advantages over bending angle retrieval. Refractivity retrieval:
1. shows generally better retrieval results in the stratosphere up to about $35 \mathrm{~km}$ in our simplified setup, caused by the introduced smoothing within the bending angle to refractivity processing

2. allows faster processing, since the involved forward model is less CPU intensive, and analytical derivatives of the forward model are much easier to calculate when compared to bending angles

3. do not terminate when the a priori data shows critical refraction at one level, where bending angles would require special treatment

Nevertheless, the retrieval from bending angles shows slightly better improvements in the upper stratosphere. It is also not affected in the retrieval by noise at higher altitudes, where refractivity profiles need to be smoothed.

Acknowledgements. This work was supported by an Internal Government Study from the Integrated Program Office of the National Polar-orbiting Operational Environmental Satellite System (NPOESS) and by the Office of Naval Research (ONR). A. von Engeln was partly funded by the German Federal Ministry of Education and Research (BMBF), within the AFO2000 project UTH-MOS, grant 07ATC04. This is also a contribution to COST Action 723 'Data Exploitation and Modeling for the Upper Troposphere and Lower Stratosphere'. The authors wish to thank N. Kreitz (ECMWF, Reading, UK) and J. Meyer-Arnek (Institute of Environmental Physics, University of Bremen, Germany) for support with the ECMWF data extraction, U. Foelsche and J. Ramsauer (Institute for Geophysics, Astrophysics, and Meteorology, University of Graz, Austria) for help with the EGOPS tool, and G. Kirchengast (Institute for Geophysics, Astrophysics, and Meteorology, University of Graz, Austria) for helpful comments on the manuscript.

Edited by: D. McKenna

\section{References}

Bevis, M., Businger, S., Herring, T., Rocken, C., Anthes, R., and Ware, R.: GPS meteorology: Remote sensing of atmospheric water vapor using the Global Positioning System, J. Geophys. Res., 97, 15 787-15 801, 1992.

Borbas, E., Menzel, W., Li, J., and Woolf, H.: Combining radio occultation refractivities and IR/MW radiances to derive temperature and moisture profiles: A simulation study plus early results using CHAMP and ATOVS, J. Geophys. Res., 108, ACL 9-1 to ACL 9-15, 2003.

Collard, A. and Healy, S.: The combined impact of future spacebased atmospheric sounding instruments on numerical weatherprediction analysis fields: A simulation study, Q. J. R. Meteorol Soc., 129, 2741-2760, 2003.

ESA/EUMETSAT: The GRAS instrument on METOP, ESA/EUMETSAT Rep. (ESA No.VR/3021/PI, EUM No. EPS/MIS/IN/9), 38 p., ESA/ESTEC, Noordwijk, Netherlands, 1998. 
Fischbach, F.: A satellite method for temperature and pressure below 24 km, Bull. Am. Meteorol. Soc., 9, 528-532, 1965.

Fjeldbo, G., Kliore, A., and Eshleman, V.: The neutral atmosphere of Venus as studied with the Mariner V radio occultation experiments, Astron. J., 76, 123-140, 1971.

Gobiet, A., Kirchengast, G., Wickert, J., Retscher, C., Wang, D.Y., and Hauchecorne, A.: Evaluation of Stratospheric Radio Occultation Retrieval Using Data from CHAMP, MIPAS, GOMOS, and ECMWF Analysis Fields, in: Proc. 2. CHAMP Science Meeting, Springer Series, 531-536, 2004.

Gorbunov, M. and Lauritsen, K.: Analysis of wave fields by Fourier integral operators and their application for radio occultations, Radio Sci., 39, RS4010, doi:10.1029/2003RS002971, 2004.

Hajj, G., de la Torre Juarez, M., Iijima, B., Kursinski, E., Mannucci, A., and Yunck, T.: GPS radio occultations coming of age: spacecraft launches add two new instruments for climate monitoring, EOS, Transactions, AGU, 83, 37, 2002.

Healy, S.: Radio occultation bending angle and impact parameter errors caused by horizontal refractive index gradients in the troposphere: A simulation study, J. Geophys. Res., 106, $11875-$ $11889,2001$.

Healy, S. and Eyre, J.: Retrieving temperature, water vapour and surface pressure information from refractive-index profiles derived by radio occultation: A simulation study, Q. J. R. Meteorol. Soc., 126, 1,661-1,683, 2000.

Hedin, A.: MSIS-86 thermosphere model, J. Geophys. Res., 92, 4649-4662, 1987.

Hedin, A.: Extension of the MSIS thermosphere model into the middle and lower amosphere, J. Geophys. Res., 96, 1159-1172, 1991.

Jakob, C., Andersson, E., Beljaars, A., Buizza, R., Fisher, M., Gerard, E., Ghelli, A., Janssen, P., Kelly, G., McNally, A., Miller, M., Simmons, A., Teixeira, J., and Viterbo, P.: The IFS cycle CY21R4 made operational in October 1999, ECMWF Newsletter No 87, pp. 2-9, 2000.

Jensen, A., Lohmann, M., Benzon, H., and Nielsen, A.: Full Spectrum Inversion of radio occultation signals, Radio Sci., 38, 6-16-15, 2003.

Kirchengast, G.: End-to-end GNSS Occultation Performance Simulator (EGOPS) overview and exemplary applications, Wissenschaftl. Ber. No. 2/1998, 138 pp., Inst. for Meteorol. and Geophys., Univ. of Graz, Austria, 1998.

Kirchengast, G., Fritzer, J., and Ramsauer, J.: End-to-end GNSS Occultation Performance Simulator version 4 (EGOPS4) software user manual, Techn. Rep. ESA/ESTEC-3/2002, 472 pp., Inst. for Geophys., Astrophys., and Meteorol., Univ. of Graz, Austria, 2002.

Kuo, Y., Sokolovskiy, S., Anthes, R., and Vandenberghe, V.: Assimilation of GPS radio occultation data for Numerical Weather Prediction, Terrestial, Atmospheric and Oceanic Science, 11, 157186, 2000.

Kursinski, E., Hajj, G., Schofield, J., Linfield, R., and Hardy, K.: Observing Earth's atmosphere with radio occultation measurements using GPS, J. Geophys. Res., 102, 23 429-23 465, 1997.

Kursinski, E., Healy, S., and Romans, L.: Initial results of combining GPS occultations with ECMWF global analyses within a 1DVar framework, Earth Planets Space, 52, 885-892, 2000.

Liu, H. and Zou, X.: Improvements to a GPS radio occultation raytracing model and their impacts on assimilation of bending angle,
J. Geophys. Res., 108, 4548, doi:10.1029/2002JD003160, 2003.

Liu, H., Zou, X., Shao, H., Anthes, R., Chang, J., Tseng, J., and Wang, B.: Impact of 837 GPS/MET bending angles on assimilation and forecasts for the period June 20-30, 1995, J. Geophys. Res., 106, 31 771-31 786, 2001.

Miller, M.: Resolution studies, ECMWF technical memorandum No. 299, ECMWF, Reading, England, 1999.

Palmer, P. and Barnett, J.: Application of an optimal estimation inverse method to GPS/MET bending angle observations, J. Geophys. Res., 106, 17 147-17 160, 2001.

Palmer, P., Barnett, J., Eyre, J., and Healy, S.: A non-linear optimal estimation inverse method for radio occultation measurements of temperature, humidity, and surface pressure, J. Geophys. Res., 105, 17 513-17 526, 2000.

Poli, P.: Advanced observation operators for GPS radio occultation. Part II: A fast atmospheric refractivity gradient operator (FARGO) for calculating bending angles and refractivity, simulations and validation with CHAMP and SAC-C, Q. J. Royal Met. Soc., 130, 2807-2825, 2004.

Poli, P., Joiner, J., and Kursinski, E.: 1DVAR analysis of temperature and humidity using GPS radio occultation refractivity data, J. Geophys. Res., 107, 4448, doi:10.1029/2001JD000935, 2002.

Rieder, M. and Kirchengast, G.: Error analysis and characterization of atmospheric profiles retrieved from GNSS occultation data, J. Geophys. Res., 106, 31 755-31 770, 2001.

Rocken, C., Anthes, R., Exner, M., Hunt, D., Sokolovskiy, S., Ware, R., Gorbunov, M., Schreiner, W., Feng, D., Herman, B., Kuo, Y.H., and Zou, X.: Analysis and validation of GPS/MET data in the neutral atmosphere, J. Geophys. Res., 102, 29 849-29 866, 1997.

Rodgers, C.: Inverse Methods for Atmospheric Sounding: Theory and Practise, vol. 2 of Series on Atmospheric, Oceanic and Planetary Physics, World Scientific Publ., 2000.

Smith, E. and Weintraub, S.: The constants in the equation for atmospheric refractive index at radio frequencies, Proceedings of the I.R.E., 41, 1035-1037, 1953.

Steiner, A. and Kirchengast, G.: Error analysis for GNSS radio occultation data based on ensembles of profiles from end-to-end simulations, J. Geophys. Res., accepted, 2005.

Syndergaard, S.: Modeling the impact of Earth's oblateness on the retrieval of temperature and pressure profiles from limb sounding, J. Atmos. Terr. Phys., 60, 171-180, 1998.

Syndergaard, S., Flittner, D., Kursinski, E., Feng, D., Herman, B., and Ward, D.: Simulating the influence of horizontal gradients on retrieved profiles from ATOMS occultation measurements a promising approach for data assimilation, in: Occultations for Probing Atmosphere and Climate - Science from the OPAC-1 Workshop, pp. 221-232, 2004.

Teixeira, J.: The impact of increased boundary layer vertical resolution on the ECMWF forecast system, ECMWF technical memorandum No. 268, ECMWF, Reading, England, 55 pp., 1999.

von Engeln, A., Bühler, S., Kirchengast, G., and Künzi, K.: Temperature Profile Retrieval from Surface to Mesopause by combining GNSS Radio Occultation and passive microwave Limb Sounder Data, Geophys. Res. Lett., 28, 775-778, 2001.

von Engeln, A., Nedoluha, G., Kirchengast, G., and Bühler, S. A.: One-dimensional variational (1-D Var) retrieval of temperature, water vapor, and a reference pressure from radio occultation measurements: A sensitivity analysis, J. Geophys. Res., 108, ACL 7-1 to ACL 7-13, 2003a. 
von Engeln, A., Nedoluha, G., and Teixeira, J.: An analysis of the frequency and distribution of ducting events in simulated radio occultation measurements based on ECMWF fields, J. Geophys. Res., 108, ACL 3-1 to ACL 3-12, 2003b.

Wickert, J., Gobiet, A., Beyerle, G., Foelsche, U., Kirchengast, G., Schmidt, T., and Steiner, A.: GPS radio occultation with CHAMP: Comparison of inversion results from GFZ Potsdam and IGAM Graz, in: Proc. 2. CHAMP Science Meeting, Springer Series, 525-530, 2004.

Wickert, J., Beyerle, G., König, R., Heise, S., Grunwaldt, L., Michalak, G., Reigber, C., and Schmidt, T.: GPS radio occultation with CHAMP and GRACE: A first look at a new and promising satellite configuration for global atmospheric sounding, Ann. Geophys., 23, 653-658, 2005,

SRef-ID: 1432-0576/ag/2005-23-653.
Zou, X., , Wang, B., Liu, H., Anthes, R., Matsumura, T., and Zhu, Y.: Use of GPS/MET refraction angles in three-dimensional variational analysis, Q. J. R. Meteorol. Soc., 126, 3013-3040, 2000.

Zou, X., Liu, H., and Anthes, R.: A statistical estimate of errors in the calculation of radio-occultation bending angles caused by a 2D approximation of ray tracing and the assumption of spherical symmetry of the atmosphere, J. Atmospheric and Oceanic Technology, 19, 51-64, 2002.

Zuffada, C., Hajj, G., and Kursinski, E.: A novel approach to atmospheric profiling with a mountain-based or airborne GPS receiver, J. Geophys. Res., 104, 24 435-24 447, 1999. 